\title{
Disruption of the structure of larval foraging behaviour in interspecific hybrids in Drosophila
}

\author{
R. GODOY-HERRERA†, B. BURNET* + K. CONNOLLY§, D. GREY\# \& I. WEIR \# \\ tDepartamento de Biologiá Celular y Genética, Universidad de Chile, Casilla 6556, Santiago 7, Chile, †Department of \\ Molecular Biology and Biotechnology, sDepartment of Psychology and \# Department of Probability and Statistics, \\ University of Sheffield, Sheffield S10 2UH, U.K.
}

\begin{abstract}
Larval foraging behaviour in two sibling species, Drosophila pavani and Irosophila gaucha belonging to the mesophragmatica species group, is described. Interspecific hybrid larvae derived from both reciprocal crosses of the parent species show disruption in the organization of their behaviour, leading to lower mean feeding rates. This, together with interactions involving biotic residues, described elsewhere, is likely under competitive conditions to contribute to reduced fitness of the hybrids relative to the parent species.
\end{abstract}

Keywords: Drosophila, foraging, interspecific hybrids, larval behaviour.

\section{Introduction}

The mesophragmatica group includes at least nine species of South American Drosophila. They are predominantly Andean in their distribution (Brncic \& Koref-Santibanez, 1957; Val et al., 1981). Drosophila pavani occurs in central Chile whereas D. gaucha, the most widely distributed member of the group, occurs in Perú, Bolivia, Southern Brazil, Uruguay and Argentina. These two species, which are allopatric for virtually all of their respective ranges, are known to be sympatric at a point on the Eastern slope of the Andes (Brncic, 1970). The two species are similar in adult morphology.

Chromosomal and genetic distance based dendrograms support the view that they form a pair of closely related sibling species within the group (MacIntyre \& Collier, 1986). D. pavani and D. gaucha mate freely under laboratory conditions giving rise to viable interspecific hybrids of both sexes, although hybrids from both reciprocal crosses are sterile (Brncic \& KorefSantibanez, 1957; Koref-Santibanez, 1964).

Brncic \& Budnik (1974) observed that the viability of pre-adult stages of the interspecific hybrid progeny of $D$. pavani and $D$. gaucha is significantly reduced in the presence of the parental species. They suggested

*Correspondence: Department of Molecular Biology and Biotechnology, University of Sheffield, P.O. Box 594, Firth Court, Western Bank, Sheffield S 10 2UH, U.K. several possible reasons for the reduced competitive ability of the hybrids, including such factors as deleterious effects of metabolic waste products and differences in behaviour. Evidence for the former possibility was subsequently provided by Budnik \& Brncic (1975) who showed that biotic residues differentially affect pre-adult viability of the different genotype groups. The investigation reported here examines the possibility that differences in behaviour contribute to reduced competitive ability of the interspecific hybrids.

Larval behaviour is normally recorded as a series of events. The sequence of transitions formed from these events could be random or non-random indicating that the behaviour has structure. Hitherto the main techniques employed for distinguishing between these possibilities were based on the construction of transition matrices. Approaches to the detection of structure in data of this kind depended on using z-scores (Green et al., 1983), or applying a chi-squared test (Brown, 1974; Fagan \& Mankovich, 1980). For the analyses of larval behaviour reported here two different non-parametric tests, involving orderings of the sequences of events, were used to circumvent some of the problems which can arise as a consequence of zero cell entries as well as the relative sparseness of transition matrices for larval behaviour.

Beyond a demonstration that larval behaviour has structure lie other questions such as, is the structure the same for larvae of different species and does it change 
as environmental conditions change? Similar questions concerning courtship behaviour in related species have been explored using principal components analysis on transition matrices (Cobb et al., 1989). In the study reported here alternative approaches were employed to detect and compare behavioural structures (Weir, 1988). An outline of these is given together with the results of species comparisons in different environments.

\section{Materials and methods}

\section{Stocks}

Drosophila gaucha, 'Tainhas' strain, was captured in Rio Grande do Sul, Brazil. Drosophila pavani, 'Quilicura' strain was collected about $20 \mathrm{~km}$ north of Santiago in Chile. The stocks were obtained from the Department of Cellular Biology and Genetics, Faculty of Medicine, University of Chile where they are maintained by mass culture.

Females were allowed to oviposit in agar-filled watch glasses overlaid with a thin layer of live baker's yeast for a period of $4 \mathrm{~h}$. Newly hatched first instar larvae were transferred to freshly yeasted watchglasses at a standard density of 50 individuals to develop under conditions of ad libitum food provision at $25^{\circ} \mathrm{C}$. Observations on the behaviour of the larvae were made in the second instar when the larvae were $48 \mathrm{~h}$ old.

\section{Mouth hooks}

Larval mouth hooks were dissected in Ringer's solution and mounted in Berlese fluid for examination.

\section{Behavioural observations}

Observations of behaviour were made using a binocular stereomicroscope with standardized cold illumination. A single larva was transferred to a $10 \mathrm{~cm}$ diameter Petri dish containing a uniform layer of 3 per cent water-agar gel, without addition, or overlaid with fresh live baker's yeast suspended in distilled water ( 48 per cent weight/volume). This was renewed for every larva to maintain uniform conditions. All observations were made at $25^{\circ} \mathrm{C}$.

Behaviour was recorded using a keyboard with 10 independent channels connected to a microcomputer. This provided a continuous record of the occurrence and sequence of key presses and a transition matrix of behavioural states. A transition denotes a change of state from some preceding behaviour to a succeeding behaviour. The behaviours recorded were: feeding (scooping with the mouth hooks, actuated by the cephalopharyngeal apparatus), locomotion, bending, turning, retreating rearing and retracting. Each behaviour was defined according to the description given by Green et al. (1983).

Larvae were transferred to the observation dish using a moist sable brush. They respond to this physical contact by contracting and remaining motionless (freezing). Behaviours were observed and recorded over a 5 min period from the time the larva relaxed. Locomotion was recorded by making a single key press for each wave of segmental contraction originating at the anal end and terminating at the cephalic end of the larval body. The feeding key was activated during each sustained period of rhythmic scooping by the larval mouth hooks.

To control for age and environmental variables across recording sessions, larvae from each genotype group were observed in strict rotation, an equal number of larvae from each group being observed during each session.

\section{Analysis of behaviour sequences}

Detection of structure. The sequence of behavioural events for a particular larva can be considered as a ranking from the first to the last event recorded. The Kruskal-Wallis test was used to examine the distribution of states along the sequence. The null hypothesis being that events occur in a random order in the sequence.

For each larva the criterion applied was a difference significant at the $P=0.05$ level. As larvae were observed only once, each test is independent. Consequently, significance of an individual test can be regarded as a success in a trial where the probability of success is 0.05 . The number of successes in a group of larvae was then compared using the binomial distribution to evaluate overall significance for a species group or condition.

The Runs test for a particular state in a sequence was calculated by considering all other states as nonobservations. The number of runs was counted and, for a null hypothesis of randomness, the mean, variance and normal approximation statistic were computed. This was then repeated for each distinct state in the sequence.

To obtain an overall test for a larva at the $P=0.05$ level of significance, the non-independence of these tests was overcome by dividing the significance level of a Runs test by the number of tests carried out for the larva and then observing if at least one test was significant at this level. An overall test for a species group was carried out using the binomial distribution. 


\section{Comparisons of structure}

Comparisons of behavioural organization in the two species and their hybrids were made in three ways.

The Cell test. This consists of a non-parametric comparison of cell entries of transition matrices each of the order $r \times r$. Each corresponding cell was treated separately from all other entries and a Wilcoxon Rank Sum test performed on the two sets of numbers for that cell (Siegel, 1956). The test was repeated for each of the $r \times r$ cells for the two sets of matrices. As these tests are not independent, an overall test for the two sets was achieved by dividing the $P=0.05$ significance level by the total number of cells in a matrix and observing if at least one cell test exceeded this level. A problem can arise here if some comparisons have inherent differences. For example, feeding movements occur rarely on agar while rearing is virtually absent on yeast. This problem was solved by reducing the sequences to common frequent transitions and recomputing the matrices. A further problem is that the test does not take account of varying total numbers of transitions between larvae.

The Proportions test. This takes account of the varying total number of transitions between genotype groups or environments. Matrices were reduced to common frequent transitions and then calculated for each larva. A Wilcoxon Rank Sum test was computed on the proportions in the two sets of sequences. Again, a divided significance level was used for the overall significance test.

The Log-Linear model. We cannot investigate the stationarity of processes in our data because transitions for individual larvae do not coincide. Several observations at the same transition times would be required for this. Preliminary analysis of 'length of runs', 'before and after runs' matrices and 'before and after states' matrices provided evidence incompatible with the assumption of a first-order Markov model. The test used for further examination of the orders followed that of Bishop et al. (1975) using the pooled data for each species or environment and assuming stationarity.

A computational package GLIM was used for calculating the log-likelihood statistics with appropriate degrees of freedom. Sequences were found to be most successfully modelled by ignoring repeated events. Comparisons of sorts of sequences were made using the common frequent states and taking account of any differences in Markov orders between genotype groups. Full details of the analyses are given by Weir (1988).

\section{Results}

\section{Mouth hook morphology}

Figure 1 illustrates in silhouette the mouth hooks of second instar larvae which are similar in D. gaucha and $D$. pavani, allowing for some individual variation within species especially in the shape of the ventral process. There are four to six acute prominent teeth. The morphology of the mouth hooks remains essentially the same after the ecdysis to third instar, apart from an increase in size, and corresponds to type $\mathrm{C}$ of Okada (1968). Species with mouth hook morphologies of this type tend to be associated with food substrates of a more fibrous kind, including fungi (Okada, 1963).

\section{Foraging on agar}

Larvae crawl over a moist agar surface searching for food and the pattern of foraging behaviour of the two species is different (Table 1).

Second instar larvae of both species raise their heads above the substrate occasionally (Green et al., 1983). Such rearing responses may be concerned with detecting changes in olfactory stimuli and humidity. Rearing

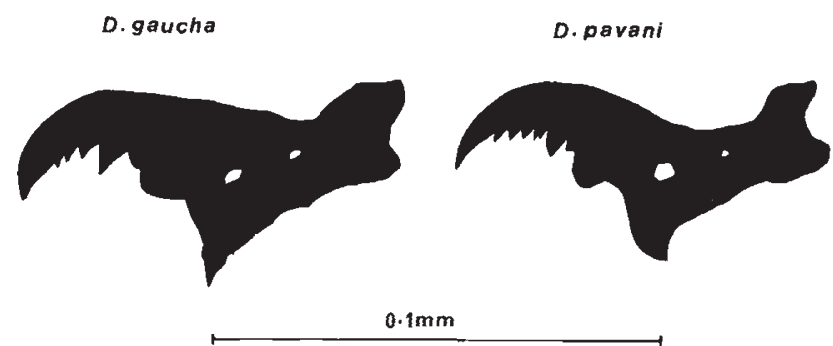

Fig. 1 Second instar larval mouth hooks of D. gaucha and D. pavani.

Table 1 Behaviour of larvae of the sibling species $D$. gaucha and $D$. pavani on a moist agar gel without food

\begin{tabular}{lcc}
\hline & D. gaucha & D. pavani \\
\cline { 1 - 2 } Locomotion & $78.6 \pm 9.3$ & $96.6 \pm 9.1$ \\
Feeding bouts & $0.40 \pm 0.28$ & 0 \\
Bending & $38.5 \pm 3.85$ & $29.3 \pm 1.93$ \\
Turning & $1.05 \pm 0.30$ & $9.85 \pm 1.25$ \\
Retreating & $2.20 \pm 0.64$ & $9.60 \pm 1.62$ \\
Retracting & $1.65 \pm 0.42$ & $4.95 \pm 0.76$ \\
Rearing & $15.3 \pm 2.2$ & $12.7 \pm 2.2$ \\
\hline
\end{tabular}

Values are the mean \pm S.E. of the number of occurrences per individual during an observation period of $5 \mathrm{~min}$. 
was not observed when larvae were surrounded by liquid. Locomotor activity tends to be higher in pavani than in gaucha but it is directed in a different manner. $D$. gaucha larvae tend to progress in a straight path over the substrate bending their heads to either side without change in orientation of the body axis. D. pavani larvae exhibited a lower rate of bending $(P<0.002)$ but had a much higher frequency of turning $(P<0.01)$. The second instar larvae of these species also differ significantly in the occurrence of retreating (reverse locomotion) and retraction. Retraction is withdrawal of the head into the body with abrupt cessation of locomotion but without contraction of the whole body, such as occurs when larvae freeze. The incidence of retracting $(P<0.01)$ and retreating $(P<0.01)$ were significantly higher in pavani. Other than occasional brief bouts of a few mouth hook movements, sustained feeding activity was absent on a smooth agar substrate.

\section{Foraging on yeast}

In yeast larval locomotor activity is not significantly different in the two species, although it tended to be higher in pavani (Table 2). Retreating occurred at a lower incidence on yeast than on agar and the two species did not differ significantly. Retracting did not occur in yeast. $D$. pavani larvae showed a significantly higher frequency of turning but bending occurred at similar rates in the two species.

Contact with a liquid substrate releases continuous feeding movements which involve scooping with the cephalopharyngeal apparatus. In a live yeast suspension the larvae show sustained feeding activity with few or no breaks throughout the observation period. There was no significant difference in the number of bouts of feeding in the two species (Table 2).

Feeding rates were measured as the mean number of cephalopharyngeal retractions per minute. Detailed analysis of the individual feeding scores, partitioned separately for male and female larvae into five separate 1 min intervals, revealed no significant difference in feeding rates between sexes, neither between minutes nor between species. In a uniform environment where food is available ad libitum the larvae of $D$. pavani and D. gaucha evidently had similar rates of feeding activity which were stable over the period of observation.

\section{Behaviour of interspecific hybrid larvae}

The mean number of occurrences of behaviours for interspecific hybrid larvae and the two parental species are given in Table 2. Analyses of variance reveal significant differences in locomotor activity between the four genotype groups $(P<0.001)$. The interspecific hybrids from both reciprocal crosses have a lower rate of locomotor activity than either parental species. Hybrid larvae from the cross $\mathrm{g} \times \mathrm{p}$ differ significantly from gaucha $(P<0.05)$, and from pavani $(P<0.01)$. The difference in locomotor activity between $\mathrm{p} \times \mathrm{g}$ hybrids and their maternal parent pavani is marginally significant at the 5 per cent level of probability but the difference from their paternal parent gaucha is not significant, neither is the difference between the two reciprocal cross means.

The rate of turning by pavani larvae is higher than that of the other genotype groups $(P<0.01)$. Interspecific hybrid larvae do not differ significantly from each other, nor from gaucha. The occurrence of bending does differ betwen genotype groups $(P<0.001)$. Hybrids of the reciprocal crosses differ from each other $(P<0.05)$ and their respective means fall beyond the range of the parental species. Hybrid larvae from the cross $\mathrm{p} \times \mathrm{g}$ show a higher mean bending rate $(P<0.05)$ than the maternal parent species (pavani) whereas larvae of the reciprocal cross $(g \times p)$ show a lower rate of bending than their maternal parent

Table 2 Behaviour of larvae of $D$. gaucha, $D$. pavani and their interspecific hybrids on live yeast suspension

\begin{tabular}{lcccc}
\hline & D. gaucha & $\mathrm{g} \times \mathrm{p}$ & $\mathrm{p} \times \mathrm{g}$ & D. pavani \\
\hline Locomotion & $97.1 \pm 5.1$ & $73.6 \pm 6.1$ & $88.6 \pm 7.5$ & $110.4 \pm 4.3$ \\
Feeding bouts & $1.6 \pm 0.38$ & $72.9 \pm 13.9$ & $41.5 \pm 8.4$ & $2.1 \pm 0.49$ \\
Bending & $23.9 \pm 1.8$ & $19.1 \pm 2.7$ & $31.3 \pm 2.3$ & $21.0 \pm 0.60$ \\
Turning & $7.9 \pm 1.7$ & $5.3 \pm 0.7$ & $8.3 \pm 1.3$ & $22.9 \pm 2.3$ \\
Retreating & $1.0 \pm 0.34$ & $0.65 \pm 0.36$ & $2.0 \pm 1.20$ & $1.2 \pm 0.46$ \\
Feeding Rate & $102.1 \pm 4.4$ & $86.6 \pm 4.3$ & $96.3 \pm 5.3$ & $110.6 \pm 5.7$ \\
\hline
\end{tabular}

Behaviour is expressed as the mean \pm S.E. number of occurrences per individual over an observation period of $5 \mathrm{~min}$. Retracting and rearing do not occur in this environment. Feeding rate is shown as the mean \pm S.E. number of cephalopharyngeal retractions per minute. 
species (gaucha) although the difference does not reach a level of statistical significance. There were no significant differences in the incidence of retreating between the genotype groups.

The most striking comparison is between larvae of the interspecific hybrid crosses and the parental species with respect to mean number of feeding bouts (Table 2). To take account of heterogeneity of variance on this measure over the four groups a Kruskal-Wallis test was used. This shows differences between groups to be significant at $P<0.001$. The feeding activity of larvae of both parent species proceeds continuously or with no more than an occasional break. Feeding by the interspecific hybrid larvae involves many bouts punctuated by breaks of variable duration. The incidence of breaks is greater in larvae from the cross $g \times p$ which consequently show the highest mean number of discrete bouts.

Although the mean feeding rate is lowest for larvae of the $g \times p$ cross, the means of the reciprocal interspecific hybrids do not differ significantly from each other. However, the mean feeding rate for $g \times p$ hybrid larvae is significantly lower than that of the gaucha maternal parent species $\langle P<0.01\rangle$. Similarly the mean feeding rate of $\mathrm{p} \times \mathrm{g}$ hybrid larvae is significantly lower than that of the pavani maternal parent species $(P<0.05)$.

\section{Detection of structure in larval behaviour}

Table 3 shows the results of testing for departures from randomness in the sequence of larval behaviours yielded by two separate non-parametric tests. Both

Table 3 Detection of structure in the behaviour of larvae of Drosophila gaucha, D. pavani and the interspecific hybrids on agar and yeast using the Kruskal-Wallis test and the Runs test

\begin{tabular}{|c|c|c|c|c|c|c|}
\hline & & \multicolumn{3}{|c|}{ Kruskal-Wallis } & \multicolumn{2}{|c|}{ Runs test } \\
\hline & & $N$ & $n$ & $P$ & $n$ & $P$ \\
\hline \multirow{2}{*}{ Agar } & gaucha & 20 & 4 & $<0.05$ & 15 & $<0.001$ \\
\hline & pavani & 20 & 14 & $<0.001$ & 16 & $<0.001$ \\
\hline \multirow{4}{*}{ Yeast } & gaucha & 20 & 8 & $<0.001$ & 19 & $<0.001$ \\
\hline & pavani & 20 & 8 & $<0.001$ & 19 & $<0.001$ \\
\hline & $g \times p$ & 20 & 7 & $<0.001$ & 17 & $<0.001$ \\
\hline & $p \times g$ & 19 & 5 & $<0.01$ & 12 & $<0.001$ \\
\hline
\end{tabular}

$N$ is the number of larvae tested and $n$ the number of individuals for which departures from a random sequence of behaviours are significant to at least the $5 \%$ level. The probability $P$ for overall significance of each genotype, using the binomial distribution, is also shown. lead to concordant conclusions: that the sequence of larval behaviour is non-random for all genotype groups in both the presence and absence of food. The tests differ with respect to the proportion of individual larvae within a group for which significant departure from randomness was detected but, even on what appears the most conservative test, Kruskal-Wallis, the evidence for structure in the sequence of larval behaviours is overwhelming.

\section{Effects of genotype and environment}

Figures 2 and 3 illustrate the behavioural organization of the parental species on agar and in yeast using ethograms. Statistical comparisons are summarized in Tables 4 and 5. On agar, with no food present, the behaviour of pavani larvae appears to be more complex than that of gaucha. The most obvious differences are in the transitions involving retracting and retreating, together with those between locomotion and turning, all of which are higher in pavani. Transitions between locomotion and bending are more frequent in gaucha. These contrasts are also evident on yeast, where feeding is the dominant activity, but transitions involving feeding occur at relatively low frequency because feeding activity occurs in long sustained bouts. Both species show a highly significant difference in the organization of their behaviour in relation to environmental change (Table 4). Species differences in behavioural organization when the larvae are on agar are significant on two of the three tests but on yeast they are significant on all the tests used (Table 5).

The organization of the behaviour of the reciprocal interspecific hybrid groups is distinguishable on only one of the three tests. The most overt difference between the ethograms is the recurrence of retreating by $\mathrm{p} \times \mathrm{g}$ larvae which also have a higher frequency of transitions between feeding and locomotion. The high frequency of short bouts of feeding activity in these hybrids underlies the high frequencies of transitions between feeding, bending and locomotion relative to the parent species.

Table 4 Comparison of the behavioural organization on agar and yeast suspension for larvae of the two sibling species

\begin{tabular}{lllll}
\hline & & Cell test & $\begin{array}{l}\text { Proportions } \\
\text { test }\end{array}$ & $\begin{array}{l}\text { Log-linear } \\
\text { test }\end{array}$ \\
\hline Agar vs. Yeast & gaucha & $P<0.001$ & $P<0.001$ & $P<0.001$ \\
pavani & $P<0.001$ & $P<0.001$ & $P<0.001$ \\
\hline
\end{tabular}

$P$ denotes the probability that behavioural structure in the two environments is the same. 
Table 5 Comparisons of the structure of larval behaviour of different genotype groups on live yeast suspension

\begin{tabular}{|c|c|c|c|c|}
\hline & & Cell test & Proportions test & Log-linear test \\
\hline \multirow[t]{4}{*}{ Agar } & gaucha vs. pavani & n.s. & $P<0.01$ & $P<0.001$ \\
\hline & gaucha vs. pavani & $P<0.001$ & $P<0.001$ & $P<0.001$ \\
\hline & gauchavs. $\mathrm{g} \times \mathrm{p}$ & $P<0.05$ & n.s. & $P<0.001$ \\
\hline & gaucha vs. $\mathrm{p} \times \mathrm{g}$ & $P<0.05$ & $P<0.05$ & n.s. \\
\hline \multirow{3}{*}{ Yeast } & pavani vs. $\mathrm{g} \times \mathrm{p}$ & $P<0.001$ & $P<0.001$ & $P<0.001$ \\
\hline & pavani vs. $\mathrm{p} \times \mathrm{g}$ & $P<0.001$ & $P<0.001$ & $P<0.001$ \\
\hline & $\mathrm{g} \times$ p vs. $\mathrm{p} \times \mathrm{g}$ & n.s. & n.s. & $P<0.001$ \\
\hline
\end{tabular}

$P$ denotes the probability that the two genotypes compared have the same behavioural organization.

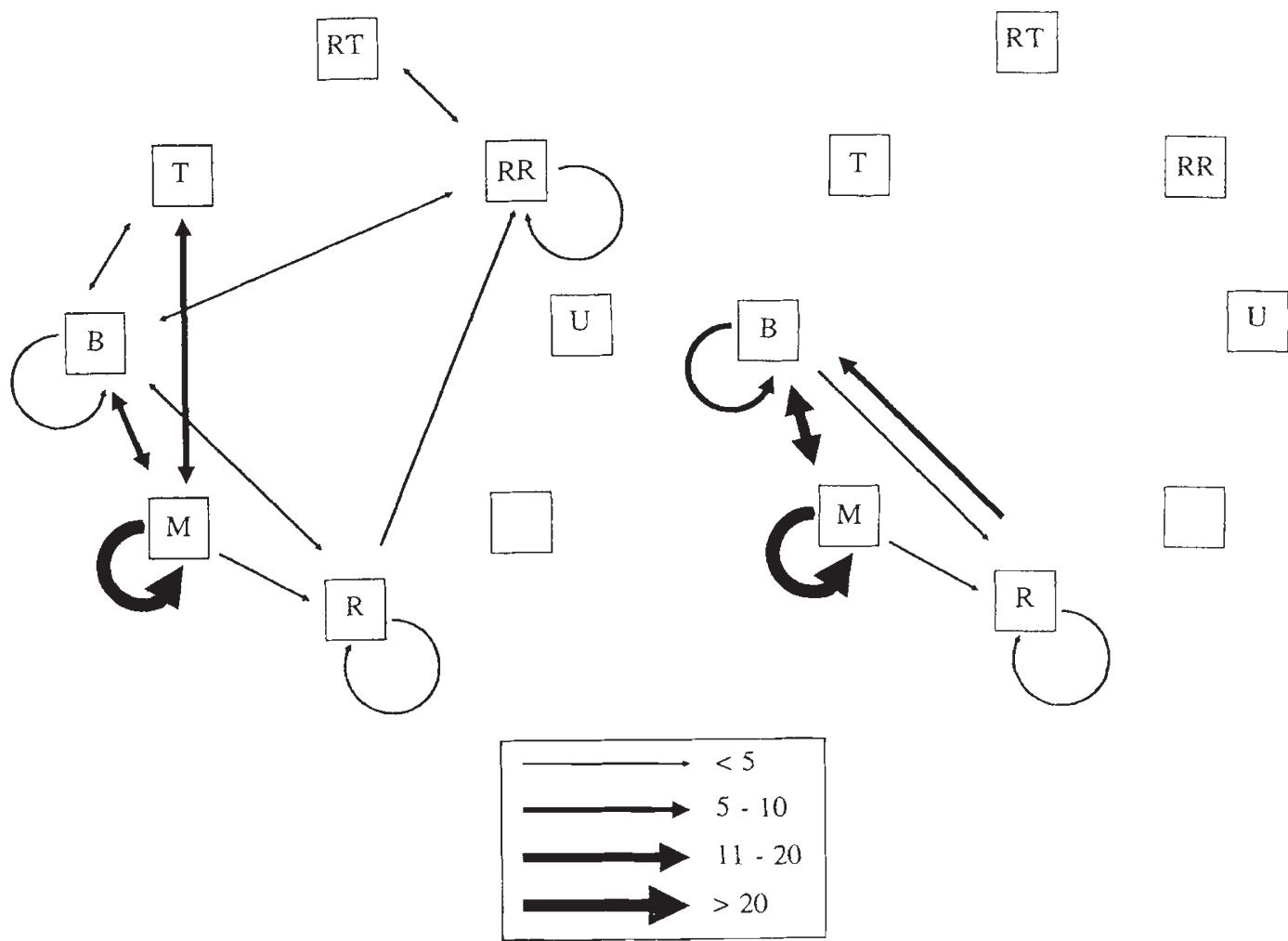

Fig. 2 Ethograms for behaviour of larvae of $D$. pavani (left) and $D$. gaucha (right) on a moist agar substrate without food. The occurrence of a behaviour and transitions between behaviours are included only if they were observed with a mean frequency of at least once per larva. Arrows indicate the direction and frequency of occurrence of transitions between behaviours. Key to behaviours: B: bend, F: feed (not indicated), M: locomotion, R: rear, RR: Retreat, RT: Retract, T: turn, U: unfreeze.

\section{Discussion}

The similarity of mouth hook morphology of $D$. pavani and $D$. gaucha implies similarity of substrate texture in their respective larval resource niches but there appears to have been a divergence in foraging behaviour between them. Foraging behaviour of Drosophila melanogaster larvae has been analysed by Sokolowski
(1980) who has distinguished alternative strategies known as rover and sitter. Behavioural polymorphism for these characters appears to be present in laboratory and wild populations of this species (Sokolowski et al., 1986). In view of the potential importance of larval foraging behaviour in relation to fitness, especially in mixed species associations, an extension of compara- 


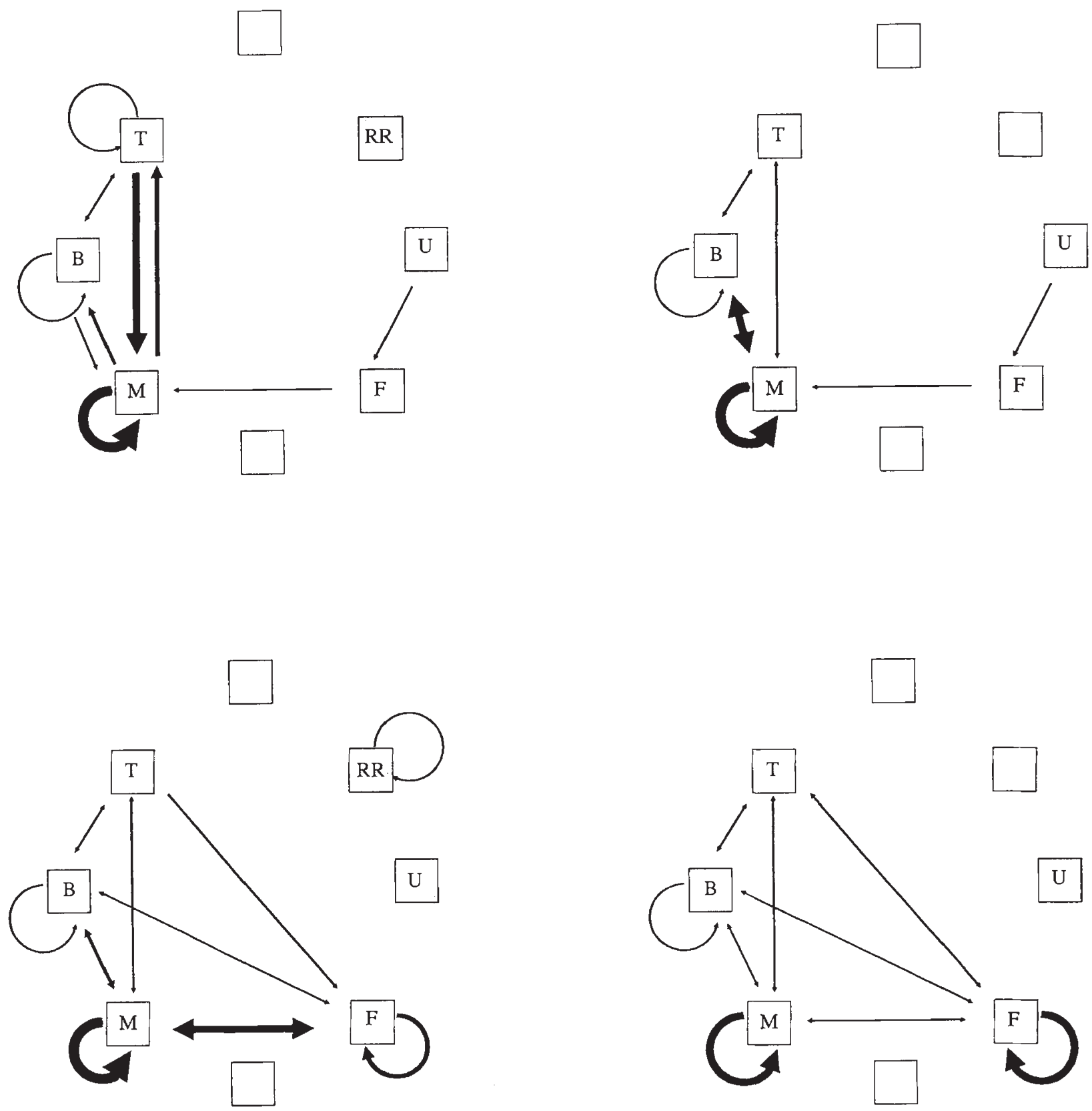

Fig. 3 Ethograms for larval behaviour in the presence of food. Parental species: D. pavani (upper left), D. gaucha (upper right). Interspecific hybrids: pavani $\times$ gaucha (lower left), gaucha $\times$ pavani (lower right). Key to behaviours as in Fig. 2 .

tive studies to a range of species within the genus is desirable.

During the trophic stages of development $D$. pavani larvae appear to dig deeper and feed at a lower level in the substrate than D. gaucha (Godoy-Herrera, 1986). Because of differences in measurement criteria, the surface foraging activity of $D$. pavani and D. gaucha cannot precisely be assigned to the rover or sitter categories as characterized by Sokolowski (1980). However, D. gaucha larvae traverse a straighter path over greater distances from their starting point, which suggests rover-like behaviour. D. pavani, with their tenfold higher turning rate pursue a convoluted path within a more narrowly circumscribed area, suggesting sitter-like behaviour. The possibility that there is also behavioural polymorphism for foraging behaviour within these two mesophragmatica species must await further investigations.

The two species appear to have retained quite similar rates of cephalopharyngeal retraction, in sustained 
bouts of feeding activity, punctuated by few and transitory breaks. Feeding activity in both species is lower, and their locomotor activity higher, than those of $D$. melanogaster and D. simulans (Green et al., 1983; Godoy-Herrera et al., 1984).

Disruption of the normal pattern and rate of feeding activity, in both reciprocal cross interspecific hybrids, implies substantial divergence of the genetic organization of feeding activity between the parental species. The evidence suggests the existence of mutually coadapted gene systems controlling feeding behaviour within each species (Wallace, 1991).

Larval feeding rate in D. melanogaster is one of a set of inter-related variables affecting survival, development rate and body size of the adult insect. Consequently it has an important bearing on fitness, especially under conditions of scramble type competition (Burnet et al., 1977). Interactions between these variables are likely to be similar, although varying in detail, in other species within the genus. Differences in foraging behaviour may have little influence on viability when food is abundant but, when food supply is a limiting resource, the superior competitor in a mixed species or mixed genotype association is expected to be the one with superior foraging behaviour. The interspecific hybrid larvae resulting from cross matings between $D$. pavani and $D$. gaucha are likely therefore to be at a disadvantage when in competition for limited resources with either of the parental species on account of their lower feeding rates. Brncic \& Budnik (1974) have already shown that the viability of these interspecific hybrids is reduced under overcrowded conditions when the parental species are also present. The outcome of competition is, however, affected by conditioning of the food medium by the biotic residues produced by the different genotypes. The metabolic waste products of $D$. pavani, D. gaucha, and their hybrids, reduce the viability of $D$. pavani and the hybrid larvae, whereas survival of $D$. gaucha larvae is affected only by its own biotic residues (Budnik \& Brncic, 1975). These observations, together with those presented here, indicate that in mixed associations the interspecific hybrid larvae suffer a double disadvantage which is both physiological and behavioural.

\section{Acknowledgements}

Thanks are due to the Royal Society and Commission Nacional de Investigacion Cientifica y Tecnologica of Chile (CONICYT) for support to enable R. GodoyHerrera to work in the U.K. The work reported here is part of a programme supported by the Link scheme of the British Council which is gratefully acknowledged.

\section{References}

BISHOP, Y. M. M., FIENBERG, S. E. AND HOLlAND, F. W. 1975. Multivariate Analysis. Massachusetts Institute of Technology Press, Cambridge, MA.

BRNCIC, D. 1970. Studies on the evolutionary biology of Chilean species of Drosophila. In: Hecht, M. K. and Steere, W. C. (eds) Essays in Evolution and Genetics in Honor of Theodosius Dobzhansky, pp. 401-436. North Holland Publishing Company, Amsterdam.

BRNCIC, D. AND BUDNIK, M. 1974. Rate of development and viability of hybrids between Drosophila pavani and Drosophila gaucha under competitive conditions. Ecology, 55, 662-666.

BRNCIC, D. AND KOREF-SANTIBANEZ, s. 1957. The Mesophragmatica group of species of Drosophila. Evolution, 11, 300-310.

BROWN, M. B. 1974. Identification of the sources of significance in two-way contingency tables. Appl. Statist., 23, 405-413.

BUDNIK, M. AND BRNCIC, D. 1975. Response of Drosophila pavani, Drosophila gaucha and their hybrids to larval biotic residues. Experientia, 31, 781-782.

BURNET, B., SEWELL, S. AND BOS, M. 1977. Genetic analysis of larval feeding behaviour in Drosophila melanogaster. II. Growth relations and competition between selected lines. Genet. Res., 30, 149-162.

COBB, M., BURNET, B., BLIZARD, R. AND JALlon, J.-M. 1989. Courtship in Drosophila sechellia: its structure, functional aspects and relationship to those of other members of the Drosophila melanogaster species subgroup. J. Insect Behav., 2, 63-89.

FAGAN, R. M. AND MANKOVICH, N. J. 1980. Two-act transitions, partitioned contingency tables and the 'significant cells' problem. Anim. Behav., 28, 1017-1023.

GODOY-HERRERA, R. 1986. The development and genetics of digging behaviour in Drosophila larvae. Heredity, 56, 33-41.

GODOY-HERRERA, R., BURNET, B., CONNOLLY, K. AND GOGARTY, J. 1984. The development of locomotor activity in Drosophila melanogaster larvae. Heredity, 52, 63-75.

GREEN, C. H., BURNET, B. AND CONNOLLY, K. J. 1983. Organization and patterns of inter and intraspecific variation in the behaviour of Drosophila melanogaster larvae. Anim. Behav., 31, 282-291.

KOREF-SANTIBANEZ, s. 1964. Reproductive isolation between the sibling species Drosophila pavani and Drosophila gaucha. Evolution, 18, 245-251.

MacINTYRE, R. J. AND COLlier, G. E. 1986. Protein evolution in the genus Drosophila. In: Ashburner, M., Carson, H. L. and Thompson, J. N. (eds) The Genetics and Biology of Drosophila, vol. 3e, pp. 39-146. Academic Press, London.

OKADA, T. 1963. Caenogenetic differentiation of mouth hooks in Drosophilid larvae. Evolution, 17, 84-98.

OKADA, T. 1968. Systematic Study of the Early Stages of Drosophilidae. Bunka Zugeisha Co., Tokyo.

SIEGEL, S. 1956. Nonparametric Statistics for the Behavioral Sciences, McGraw-Hill Kogakusha, Tokyo. 
Sokolowski, M. в. 1980. Foraging strategies of Drosophila melanogaster: a chromosomal analysis. Behav. Genet., 10, 291-302.

SOKOLOWSKI, M. B., BAUER, S. S., WAI-PING, V., RODRIGUEZ, L., WONG, J. L. AND DENT, C. 1986. Ecological genetics and behaviour of Drosophila melanogaster larvae in nature. Anim. Behav., 34, 403-408.

VAL, F. C., VILELA, C. R. AND BARQUeS, M. S. 1981. Drosophilidae of the Neotropical region. In: Ashburner, M., Carson, H.
L. and Thompson, J. N. (eds) The Genetics and Biology of Drosophila, vol. 3a, pp. 123-168. Academic Press, London.

WAllaCE, B. 1991. Coadaptation revisited, J. Hered., 82, 89-95.

WEIR, I. S. 1988. Sequence Analysis of Larva Behaviour in Mutants and Species of the Fruit Fly Drosophila. M.Sc. Thesis, Department of Probability and Statistics, University of Sheffield. 